\title{
Investigação da Memória de Longo Prazo na Taxa de Câmbio no Brasil*
}

\author{
Sergio R. S. Souza ${ }^{\dagger}$, Benjamin M. Tabak ${ }^{\ddagger}$, Daniel O. Cajueiro ${ }^{\S}$
}

Sumário: 1. Introdução; 2. Metodologia; 3. Dados; 4. Resultados empíricos; 5. Conclusão.

Neste trabalho, é medida a evolução da memória de longo prazo da taxa de câmbio diária, Real contra Dólar dos Estados Unidos, no período de 1995 a 2004. Essa medição é realizada por meio da análise R/S clássica, com janela móvel de dados. O trabalho focaliza o abandono do regime de câmbio administrado em favor do de câmbio flutuante, ocorrido em 1999, identificando antipersistência da taxa de câmbio durante a vigência do primeiro regime e memória longa a partir do início da vigência do segundo regime. Mostra também evidência de memória longa para as volatilidades dos retornos das taxas analisadas.

This paper presents measures of long-range dependence in daily exchange rates of the Brazilian Real against the US Dollar, taken from 1995 to $2004 \mathrm{em}$ ploying the classical $R / S$ analysis with a rolling sample. It analyses the switch from a crawling peg exchange regime to a floating exchange regime, in 1999, finding antipersistence in the exchange rate during the first exchange regime, and long memory in the exchange rates along the second one. Also, it finds long memory for the exchange rates' volatilities along the whole period.

Palavras-chave: Palavras-chave: taxa de câmbio; dependência de longo prazo; volatilidade; Análise R/S. Códigos JEL: F31; C89.

\footnotetext{
* Os autores agradecem as valiosas sugestões e comentários de Carlos Araújo e dos pareceristas anônimos. Benjamin M. Tabak agradece a Bolsa de Produtividade em Pesquisa do CNPQ. As opiniões expressas neste artigo são de inteira responsabilidade dos autores e não refletem a opinião do Banco Central do Brasil.

†Departamento de Operação das Reservas Internacionais (Depin) do Banco Central do Brasil, SBS Quadra 3, Bloco B $5^{\circ}$ andar, Brasília - DF 70074-900, Brasil.

¥Departamento de Estudos e Pesquisas (Depep) do Banco Central do Brasil, SBS Quadra 3, Bloco B $9^{\circ}$ andar, Brasília - DF 70074900, Brasil, e-mail para correspondência: benjamin.tabak@bcb.gov.br

§Programa de Pós-Graduação em Economia - Universidade Católica de Brasília (UCB) - SGAN 916, Módulo B - Asa Norte, Brasília - DF 70790-160, Brasil
} 


\section{INTRODUÇÃO}

A possibilidade de se prever o comportamento de taxas de câmbio é tema de interesse para várias categorias de agentes do mercado. Podem se beneficiar, de diferentes formas, os formuladores e executores de políticas econômica e cambial, os hedgers e os especuladores em suas respectivas áreas de atuação. No entanto, dada a complexidade do processo que produz o comportamento desse preço, a área de estudo em questão ainda está em aberto. A complexidade deriva do fato de não se poder compreender o efeito dos fatores que determinam as taxas de câmbio a partir da avaliação isolada de cada um deles.

O estudo do comportamento da taxa de câmbio pode ser feito do ponto de vista da modelagem de processos: nesse caso, procura-se identificar um conjunto de fatores que interagem entre si para formar a taxa; em seguida, o comportamento desses fatores é modelado individualmente e em conjunto. Para a realização desse trabalho, são necessárias medições que muitas vezes não são possíveis, por exemplo, as variações na credibilidade de uma política cambial (o que se pode medir é o resultado dessa variação de credibilidade). Outra maneira de se abordar o comportamento das taxas de câmbio é estudar diretamente o seu movimento, sem a modelagem dos fatores que o causam. Nesse caso, são realizados testes para a identificação de tipos estilizados de processos que poderiam melhor se ajustar ao movimento observado de taxas. São realizadas medidas indicativas da ocorrência, na série de taxas de câmbio, do processo que está sendo testado, e em seguida, são realizados testes estatísticos, nas medidas realizadas, da ocorrência ou não desse processo.

Neste trabalho, será feita uma investigação da ocorrência de memória de longo prazo, variável no tempo, para retornos e volatilidades das taxas de câmbio entre real e dólar, seguindo a última abordagem citada, ou seja, não será estudada uma maneira de se preverem as taxas de câmbio, mas somente se os retornos e volatilidades das taxas observadas no período analisado, de abril de 1995 a outubro de 2004, apresentam memória de longo prazo - se os valores observados de retornos e volatilidades em lags distantes estão correlacionados entre si. A identificação de memória de longo prazo nas séries de retornos e volatilidades das taxas de câmbio resulta na possibilidade de melhoria das previsões que podem ser feitas por meio de modelos econométricos e que levam em conta a ocorrência de memória de longo prazo, como o ARFIMA ( $k, d, l)$ (Auto Regressive Fractionally Integrated Moving Average), proposto por Granger e Joyeux (1980) e Hosking (1981), ou o FIGARCH (Fractionally Integrated Generalized Auto Regressive Conditional Heteroskedasticity), proposto por Bollerslev et alii (1996). Será verificado, também, se a mudança do regime de câmbio administrado para flutuante provocou impacto na memória de longo prazo medida para retornos e volatilidades de taxas de câmbio.

A maioria dos trabalhos que envolveram a identificação ou a estimação de memória de longo prazo se refere a todo o conjunto de dados e fornece um único valor para todo o período da amostra. Nenhum trabalho do qual tenhamos conhecimento realizou estimação da evolução da memória de longo prazo com o tempo para taxas de câmbio de real x dólar dos EUA. Cajueiro e Tabak (2004a), Cajueiro e Tabak (2004b), Cajueiro e Tabak (2004c), Cajueiro e Tabak (2005a), Cajueiro e Tabak (2005b) e Cajueiro e Tabak (2005c) realizaram estimações da evolução temporal da memória de longo prazo, no entanto, não estudaram as taxas de câmbio entre real e dólar dos EUA. A propósito, um estudo interessante nesse contexto, e que considera o estudo de memória de longo prazo em taxas de câmbio de dólar brasileiro, foi realizado por Laurini e Portugal (2004). Nesse trabalho, são estudados os retornos de taxas de câmbio brasileiras no período de $1^{\circ}$ de julho de 1994 a 4 de janeiro de 2002, utilizando os resíduos de um processo de Markov Switching. Os autores mostram que, no caso de uma estimação estática do parâmetro, a evidência de memória de longo prazo não pode ser sustentada.

Neste trabalho, é feita a estimação da memória de longo prazo variável no tempo, para retornos e volatilidades das taxas de câmbio diárias de fechamento PTAX, por meio da análise $R / S$ clássica, de Hurst (1951) e Mandelbrot (1972), calculada com janela móvel de 504 observações e embaralhamento de dados dentro de blocos contíguos de dez observações, seguindo a abordagem empregada por Cajueiro e Tabak nos trabalhos citados acima. A estimação da evolução temporal da memória de longo prazo 
dos retornos permite a avaliação da evolução da eficiência do mercado de câmbio e a identificação da reação dessa eficiência a eventos que podem afetar o mecanismo do processo gerador de preços. Além disso, permite a melhoria na avaliação do risco associado ao ativo analisado, pois a volatilidade é uma medida importante de risco.

O trabalho está estruturado da seguinte forma: na próxima seção, são apresentadas a metodologia de estimação de memória de longo prazo utilizada e uma breve revisão teórica; na seção 3, são apresentados os dados e o contexto de onde esses dados foram extraídos; na seção 4, os resultados são apresentados; e, na seção 5, é registrada a conclusão do trabalho.

\section{METODOLOGIA}

Uma série de observações apresenta memória de longo prazo quando os valores observados em lags distantes são correlacionados entre si, ou ainda, se o efeito de um evento ocorrido em um instante pode ser detectado muitos lags depois. Especificamente, diz-se que uma série de observações, estacionária em covariância, tem memória de longo prazo se a sua função de autocovariância não for somável (ver Giraitis et alii (2003)). A memória de longo prazo é tipicamente caracterizada pelo decaimento hiperbólico da função autocovariância, definida por $\gamma_{k}=\operatorname{Cov}\left(X_{k} ; X_{0}\right)$. Esse decaimento tem taxa $c k^{2 d-1}$, com $0<d<0,5$. Ocorrendo memória de longo prazo, tem-se $\sum_{j=-\infty}^{\infty}\left|\gamma_{j}\right|=\infty$. No caso da memória de curto prazo, a função autocovariância decai de forma exponencial, tendendo a zero rapidamente. Havendo apenas memória de curto prazo, a soma das autocovariâncias tem valor finito.

A não-detecção de memória de longo prazo em uma série de observações indica que:

a) não existe um processo dinâmico envolvendo a variável observada e outros fatores (um exemplo disso seria o movimento browniano ${ }^{1}$ );

b) o processo dinâmico existe, mas tem memória curta demais para que os efeitos do estado do sistema em um instante perdurem até o instante seguinte, estabelecido de acordo com a taxa de amostragem das observações. Como pode ter havido alguma variação (choque) em variável exógena do sistema, e como tanto a reação do sistema quanto a dissipação dos efeitos são muito rápidas, o estado do sistema em um instante não guardará relação com o estado no instante posterior e, portanto, os valores registrados na série temporal estudada não guardarão relação entre si. Isso, na prática, equivale ao movimento browniano.

A avaliação da memória de longo prazo de uma série pode ser feita por meio de diversas metodologias. Entre as metodologias hoje utilizadas na identificação e quantificação de memória de longo prazo, são mais utilizadas a análise $R / S$ clássica, desenvolvida por Hurst (1951) e Mandelbrot (1972), a análise $R / S$ modificada, de Lo (1991), a estimação do parâmetro de integração fracionária por regressão espectral, ou log-periodograma, de Geweke e Porter-Hudak (1983), o estimador semi-paramétrico por log-periodograma, de Robinson (1995) e a análise $V / S$, de Giraitis et alii (2003) e Cajueiro e Tabak (2005d). Essas metodologias podem ser utilizadas sem que se tenha conhecimento dos fatores que atuam no processo gerador de preços, levando em conta apenas as séries de retornos ou volatilidades (ou resíduos dessas variáveis em relação a regressores) para as quais se deseja estimar a memória de longo prazo. Todas as metodologias descritas acima podem resultar em parâmetros de memória de longo prazo, seja o expoente de Hurst "H", ou o parâmetro de integração fracionária "d", que podem

\footnotetext{
${ }^{1}$ O movimento browniano foi observado pela primeira vez em 1828, por Robert Brown. Ele verificou que o movimento aleatório do pólen microscópico não tinha causas biológicas, como se supunha até então, mas físicas, resultantes do movimento de partículas microscópicas que colidem entre si, movidas pela energia térmica. O movimento browniano, observado ao microscópio, consiste de deslocamentos em uma direção aleatória e comprimento que pode ser descrito por valor característico. 0 termo "passeio aleatório" (random walk) é utilizado no contexto do movimento browniano. Ver Feder (1988).
} 
ser utilizados na modelagem econométrica de processos com memória de longo prazo na média, como o ARFIMA (k, d, l) (Auto Regressive Fractionally Integrated Moving Average), proposto por Granger e Joyeux (1980) e Hosking (1981).

Neste trabalho, pretende-se utilizar a análise $R / S$ clássica no cálculo do expoente de Hurst " $\mathrm{H}$ ", aplicada sobre dados embaralhados dentro de blocos contíguos ao longo de toda a série, por meio de uma janela móvel, com o intento de identificar, em séries de retornos e de volatilidades de retornos, a ocorrência de previsibilidade devida à memória de longo prazo, e sua variabilidade ao longo do tempo, nas séries de retornos e volatilidades das taxas PTAX de fechamento de real $\times$ dólar dos EUA. A metodologia utilizada neste trabalho foi inicialmente proposta por Hurst (1951), que formulou a análise $R / S$ clássica e derivou a relação empírica que fornece o que ficou conhecido como expoente de Hurst. Posteriormente, Mandelbrot e Wallis (1969b), Mandelbrot (1972) e Mandelbrot (1975) realizaram estudos para avaliar a confiabilidade da estatística $R / S$ como identificadora de previsibilidade devida a memória de longo prazo. Mandelbrot (1971) também considerou a existência de memória de longo prazo em ativos financeiros. Wallis e Matalas (1970) afirmaram que autocorrelações de curto prazo podem afetar o valor do expoente de Hurst calculado. Tendo em vista essa limitação da estatística $R / S$, Lo (1991) formulou a estatística $R / S$ modificada para evitar essa sensibilidade da estatística $\mathrm{R} / \mathrm{S}$ a correlações de curto prazo. Teverovsky et alii (1999) posteriormente verificaram que a análise R/S modificada era conservadora em relação à rejeição da hipótese nula de que não há memória de longo prazo. O conceito de expoente de Hurst variável no tempo foi introduzido por Muniandy et alii (2001). Cajueiro e Tabak (2004c) utilizaram esse conceito para avaliar a evolução da eficiência de mercados emergentes a partir de dados filtrados por processo AR-GARCH; em Cajueiro e Tabak (2004a), avaliaram o comportamento da eficiência de mercados asiáticos por meio da análise $R / S$ com janela móvel e embaralhamento de dados dentro de blocos não superpostos de 5 e de 10 observações ao longo de toda a série e, em Cajueiro e Tabak (2004b), avaliaram a volatilidade dos retornos de mercados emergentes, com relação à previsibilidade devida a memória de longo prazo utilizando a análise $R / S$ clássica sobre dados embaralhados dentro de blocos contíguos e janela móvel. Tanto a filtragem dos dados por processo AR-GARCH $(1,1)$ quanto o embaralhamento da amostra foram utilizados para evitar a distorção dos resultados causada por possíveis autocorrelações de curto prazo.

\subsection{Cálculo do Expoente de Hurst}

A análise da estatística $R / S$ foi formulada por Hurst (1951) ao estudar problemas de dimensionamento de represas, que procurou determinar qual seria a capacidade ideal do reservatório, dadas as vazões anuais a ele associadas durante o período de algumas décadas. A idéia consistia em determinar quais eram os volumes máximo e mínimo no reservatório, pois se pretendia tanto evitar o transbordamento quanto a secagem do mesmo. A diferença entre os volumes máximo e mínimo seria o range de volumes do reservatório. Além disso, era calculado o desvio-padrão das vazões de água, sendo que a estatística $R / S$ seria o resultado da divisão do range pelo desvio-padrão, que é um valor adimensional. Hurst, ao analisar essa estatística para diversos períodos de tempo dessa série de observações, descobriu que havia uma função relacionando o valor da estatística $R / S$ ao número de observações que entraram no cálculo. Mais tarde, ele verificou que essa relação também era válida para outros fenômenos naturais. Nessa relação, a estatística $R / S$ é igual à metade do número de observações elevado a um expoente "H", mais tarde denominado "Expoente de Hurst".

Mandelbrot e Wallis (1969a) e Mandelbrot (1982) verificaram que a relação empírica descoberta por Hurst exibia a mesma forma apresentada pelas séries que descrevem movimento browniano fracionário, no que se refere ao rescaled range $(\mathrm{R} / \mathrm{S})$ em função do período utilizado no cálculo $(\tau)$ e, portanto, a fórmula de Hurst era aplicável a fenômenos dotados de movimento browniano fracionário. Isso significa que a estatística $\mathrm{R} / \mathrm{S}$ e o expoente de Hurst "H" podem ser utilizados para representar as propriedades de memória de longo prazo de séries com movimento browniano fracionário. Os valores do expoente 
de Hurst obtidos dessa forma têm, então, a seguinte interpretação: $0<H<0,5$ : série antipersistente; $H=0,5$ : série apresenta random walk; $0,5<H<1$ : série persistente.

A seguir, são apresentados os cálculos a serem feitos para a determinação do expoente de Hurst associado aos retornos obtidos a partir de uma série de taxas de câmbio diárias de fechamento.

Seja $X(t)$ a taxa de câmbio de fechamento a ser estudada e $r(t)$ o retorno logarítmico dessa taxa na data, dado por :

$$
r_{t}=\ln (X(t) / X(t-1))
$$

Para a estimação da memória de longo prazo para a série de volatilidades, utiliza-se como aproximação para a volatilidade instantânea em $\mathrm{t} o$ valor absoluto de $r_{t}{ }^{2}$.

O número total de retornos da série é $N$. Para se calcular o expoente de Hurst "H ", são feitos vários cálculos de estatística $R / S$ para blocos de $\tau$ observações, sendo $\tau \leqslant N$. Para cada valor de $\tau$, a estatística $R / S$ é calculada desta forma:

a) a série de $N$ retornos é divida em $n$ blocos contíguos de $\tau$ elementos, numerados com $1 \leqslant i \leqslant n$.

Em cada bloco $\mathrm{i}$, os elementos $r_{t, i}$ são numerados com $1 \leqslant t \leqslant \quad$;

b) calcula-se a estatística $R / S$ associada ao tamanho de bloco $\tau$ :

1) calcula-se a média dos retornos de cada bloco;

$$
\bar{r}_{i}=\frac{1}{\tau} \sum_{t=1}^{\tau} r_{t, i}
$$

2) calcula-se o desvio-padrão em cada bloco:

$$
S_{i}=\left[\frac{1}{\tau} \sum_{t=1}^{\tau}\left(r_{t, i}-\bar{r}_{i}\right)^{2}\right]^{1 / 2}
$$

3) calcula-se, para cada bloco $i$, a estatística $(R / S)_{i}$ :

$$
(R / S)_{i}=\frac{1}{S_{i}}\left[\max _{1 \leqslant t \leqslant \tau} \sum_{k=1}^{t}\left(r_{k, i}-\bar{r}_{i}\right)-\min _{1 \leqslant t \leqslant \tau} \sum_{k=1}^{t}\left(r_{k, i}-\bar{r}_{i}\right)\right] ;
$$

4) calcula-se a média dos valores $(R / S)_{i}$ e se associa ao tamanho de bloco $\tau$ :

$$
(R / S)_{\tau}=\frac{1}{n} \sum_{i=1}^{n}(R / S)_{i}
$$

c) terminados os cálculos da estatística $R / S$ para diversos valores de comprimento de bloco $\tau$, obtém-se o expoente de Hurst " $H$ " da relação:

$$
(R / S)_{\tau}=(\tau / 2)^{H}
$$

1) dados os pares $(R / S)_{\tau}$ e $\tau$, obtidos em b), executa-se a regressão, conforme Mandelbrot e Wallis (1969b):

$$
\log _{10}(R / S)_{\tau}=\log _{10} \mathrm{C}+\mathrm{H} \log _{10} \tau+\text { erro; }
$$

2) o expoente de Hurst "H" é determinado com intervalo de confiança de $95 \%$.

${ }^{2}$ Cajueiro e Tabak, ao analisarem a Previsibilidade devida a memória de longo prazo de volatilidades em Cajueiro e Tabak (2005b), compararam a utilização, como aproximação de volatilidade, do retorno ao quadrado e do valor absoluto do retorno e concluíram que, apesar de a aproximação feita por meio do valor absoluto fornecer valores mais altos do expoente de Hurst ao longo do tempo, os resultados obtidos através das duas são qualitativamente iguais. 


\subsection{Avaliação da análise $\mathrm{R} / \mathrm{S}$ como método de estimação de memória de longo prazo}

A proposta da utilização do expoente de Hurst calculado a partir da estatística $R / S^{3}$ como estimador de memória de longo prazo de séries temporais tem sido avaliada por diversos estudiosos, que buscam verificar a confiabilidade dessa medida.

Entre os pontos fortes da utilização da estatística $R / S$ para estimação de memória de longo prazo, estão a superioridade desse método em relação a métodos mais convencionais, como a análise de autocorrelações, razões de variância e decomposições espectrais, de acordo com Lo (1991). A estatística $R / S$ pode identificar memória de longo prazo em séries temporais altamente afastadas da normalidade, com grande assimetria e curtose, segundo Mandelbrot e Wallis (1969a). Mandelbrot $(1972,1975)$ também relata convergência quase certeira da estatística $R / S$ para processos estocásticos com variância infinita, com evidente vantagem sobre autocorrelações e razões de variância. Além disso, Mandelbrot (1972) diz que, ao contrário da análise espectral, a análise $R / S$ pode detectar ciclos não periódicos e ciclos com período igual ou maior que o período da amostra. Lo (1991) afirma que, apesar de esses pontos fortes serem, até certo ponto, passíveis de contestação, a estatística $R / S$ clássica pode, reconhecidamente, identificar memória de longo prazo.

Dentre os pontos fracos identificados na literatura, estes parecem ser os mais citados:

a) o método é assintótico, não sendo confiável para amostras pequenas, segundo Teverovsky et alii (1999); Couillard e Davison (2005); e Feder (1988). Feder (1988) sugere ainda que esse cálculo seja feito para amostras de, pelo menos, 2500 observações extraídas de maneira confiável para que 0 resultado seja confiável;

b) os coeficientes de Hurst podem variar ao longo do tempo. Tabak e Cajueiro realizaram diversos estudos sob o pressuposto de que os processos dinâmicos subjacentes às séries eram não estacionários, realizando o cálculo do expoente de Hurst em uma janela móvel dentro das séries estudadas. Para maiores detalhes, ver Cajueiro e Tabak (2004a), Cajueiro e Tabak (2004b), Cajueiro e Tabak (2004c), Cajueiro e Tabak (2005a), Cajueiro e Tabak (2005b); Cajueiro e Tabak (2005c).

c) o método também detecta memória curta, sem diferenciá-la da memória de longo prazo. Lo (1991) propôs uma estatística $R / S$ modificada para solucionar esse problema.

Teverovsky et alii (1999) avaliaram a análise $R / S$ modificada, proposta por Lo para testar se haveria previsibilidade devida à memória de longo prazo em séries temporais geradas sinteticamente e concluíram que, apesar de a análise $R / S$ modificada representar uma melhoria sobre a análise $R / S$ clássica, ainda apresentava uma limitação: era conservadora em relação à rejeição da hipótese nula de que não ocorre previsibilidade devida a memória de longo prazo, o que significa que, se o método de Lo indicar que não há evidências de previsibilidade devida a memória de longo prazo, deve-se continuar investigando. Assim sendo, Teverovsky, Taqqu e Willinger recomendam que esse método de análise seja empregado juntamente com outras ferramentas para a verificação desse tipo de previsibilidade.

Tendo em vista essa limitação, Cajueiro e Tabak propuseram que a série a ser testada fosse filtrada por um processo $\mathrm{AR}(1)$-GARCH$(1,1)^{4}$ e os resíduos fossem submetidos à análise $R / S$ clássica (equações 4 e 6). A filtragem é feita para se extrair dos elementos da série a parcela devida à ocorrência de memória curta e à variação das volatilidades dos retornos. Se o expoente de Hurst for calculado para retornos não ajustados em relação à volatilidade, a previsibilidade encontrada pode ser devida a efeitos originados pela volatilidade deles, que são reconhecidamente persistentes em séries financeiras (2004).

\footnotetext{
3 O expoente de Hurst também pode ser calculado por meio da DFA (Detrended Fluctuation Analysis), que está fora do escopo deste trabalho. Cajueiro e Tabak (2005a) utilizaram essa técnica para estudar o mercado brasileiro de ações. Essa técnica foi desenvolvida independentemente nos trabalhos de Moreira et alii (1994) e de Peng et alii (1994).

${ }^{4}$ Generalized Auto Regressive Conditional Heteroskedastic.
} 
Outras maneiras de contornar a sensibilidade da análise $R / S$ a autocorrelações de curto prazo poderiam ser estas:

a) subdividir a série em blocos de 5, 10 ou 20 observações não sobrepostas e embaralhar cada bloco de forma aleatória, com a finalidade de destruir a estrutura de autocorrelações dentro desses blocos. Isso foi feito pela primeira vez, no contexto da identificação de memória de longo prazo, por Erramilli et alii (1996). Cajueiro e Tabak têm utilizado essa técnica em diversos estudos Cajueiro e Tabak (2004a), Cajueiro e Tabak (2005a) e Cajueiro e Tabak (2005b). Ao estudar a memória de longo prazo das volatilidades observadas nos mercados de ações de países emergentes, eles verificaram que os resultados obtidos eram robustos em relação à escolha de blocos de 5, 15, 20 e 30 observações. Nessa metodologia, a série a ser analisada é embaralhada e depois é aplicada sobre ela a análise $R / S$ clássica;

b) agregar os dados de blocos não superpostos da série e submeter o resultado dessa agregação à análise $R / S$ clássica. Nesse caso, a série a ser analisada é dividida em blocos de 5 , e tira-se a média de cada bloco, o que torna a série mais próxima da normalidade. Isso foi feito por Cajueiro e Tabak (2005a), tendo sido usado antes por Taqqu et alii (1995).

Neste trabalho, é utilizada a metodologia de embaralhamento dos dados da série, pois essa metodologia não se baseia em hipóteses restritivas em relação à volatilidade (filtro GARCH), nem reduz o número de observações a ser informado para a análise $R / S$, que traz prejuízo pelo fato de o método exigir grande número de observações.

Uma última consideração em relação à avaliação da análise $\mathrm{R} / \mathrm{S}$ clássica como método de estimação de memória de longo prazo é a questão das incertezas envolvendo o cálculo do valor do expoente de Hurst.

Em primeiro lugar, o cálculo do expoente de Hurst por meio da análise $R / S$ clássica é assintótico, o que resulta em erro, o qual será maior quanto menor for o tamanho da amostra submetida à análise. A análise $R / S$ clássica não prevê correção para a estatística $R / S$ no caso de amostras pequenas. Para tratar essa necessidade, Couillard e Davison (2005) propuseram correção para o expoente de Hurst calculado por meio da estatística $R / S$, tendo em vista o tamanho da amostra. Definiram também um cálculo para o desvio-padrão do expoente de Hurst calculado por esse novo método. Este trabalho não utiliza essa correção, mas procurará levar em conta a possibilidade de ocorrência desse tipo de distorção nas análises.

Além disso, a estatística $R / S$ clássica não tem distribuição conhecida sob a hipótese nula de não haver memória de longo prazo, de acordo com Crato e Ray (2000).

Outra fonte de incerteza na metodologia de cálculo do expoente de Hurst utilizada neste trabalho resulta de sua obtenção por meio de uma regressão, a partir dos cálculos de estatística $R / S$ média, feitos para cada comprimento de bloco no qual a amostra é dividida (ver equações 2 a 7). Dado que o valor de $H$ é obtido dessa regressão, calcula-se também o intervalo de confiança de $95 \%$ para esse valor. Para fins de identificação de memória de longo prazo, se o valor de $H=0,5$ estiver dentro do intervalo de confiança, não se poderá afirmar que a série possui memória de longo prazo.

\section{DADOS}

Os dados utilizados neste trabalho são as taxas diárias de fechamento de dólar PTAX, expressas em reais, divulgadas pelo Banco Central, no período de 3 de abril de 1995 a 6 de outubro de 2004. Os dados foram obtidos da Bloomberg. A Tabela 1 apresenta um resumo da série analisada.

A Figura 1 apresenta o gráfico das taxas de câmbio estudadas.

A partir da série de taxas de câmbio acima, são obtidas as séries que serão analisadas: i) retornos: $r_{t} \ln (X(t) / X(t-1))$, sendo $X_{t}$ e $X_{t-1}$ as taxas de câmbio de fechamento nas datas $t$ e $t-1$; e ii) 
Tabela 1 - Série analisada - Dados diários - Cotações de fechamento PTAX

\begin{tabular}{ccccc}
\hline Série & Unidade & País & Período de/até & Observações \\
\hline Dólar EUA PTAX & R\$/US\$ & Brasil & $4 / 4 / 1995$ a 7/10/2004 & 2482 \\
\hline
\end{tabular}

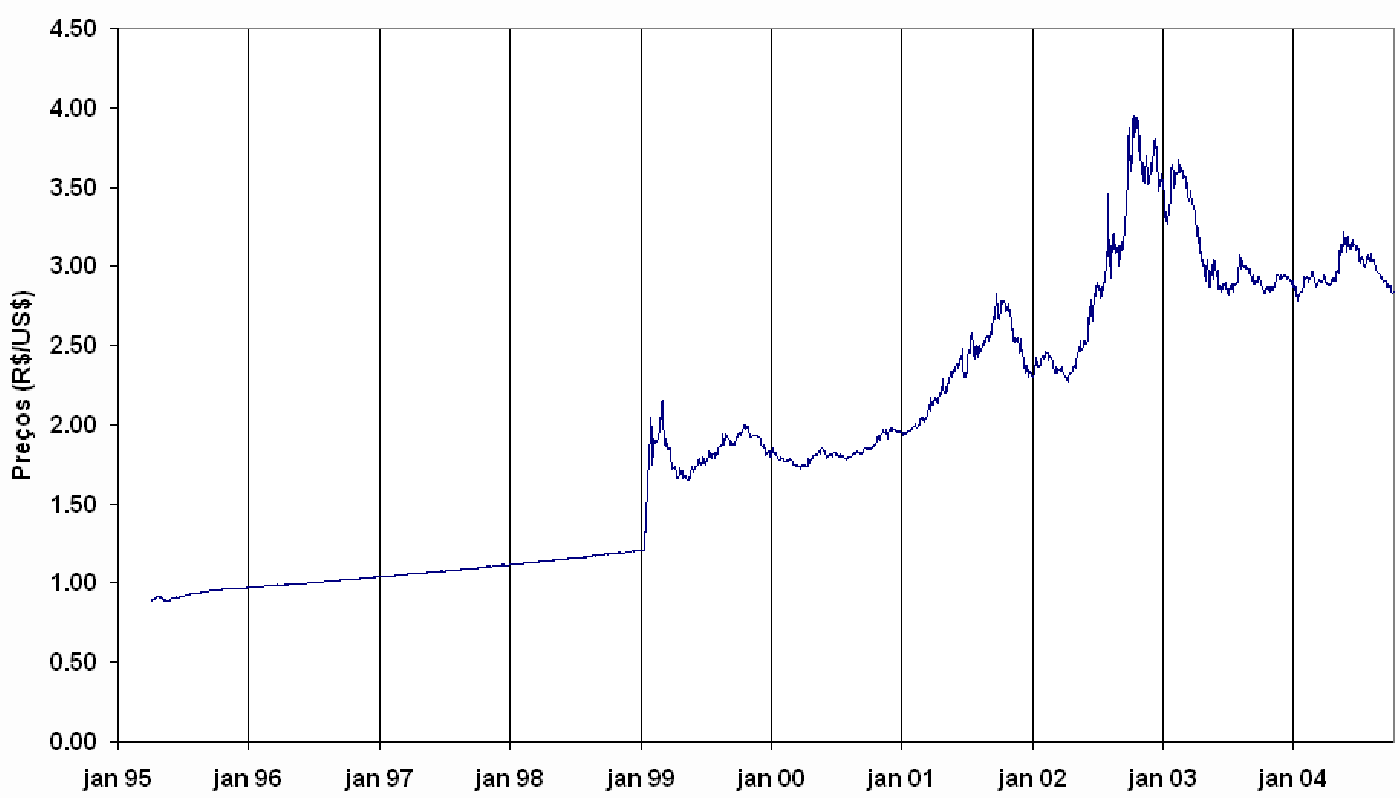

Figura 1 - Taxas de fechamento PTAX do dólar dos EUA, em reais

volatilidades: $v_{t}=$ valor absoluto de $r_{t}$, as quais serão analisadas. Devido ao fato de autocorrelações de curto prazo poderem afetar o valor do expoente de Hurst a ser calculado por meio da análise $R / S$ clássica, é feito embaralhamento de dados das amostras de retornos e volatilidades a serem estudadas, dentro de blocos contíguos de dez observações antes da realização dos cálculos, com a finalidade de destruir as autocorrelações de curto prazo porventura existentes.

A caracterização das séries de log-retornos e log-volatilidades obtidas acima é apresentada na Tabela 2. Os gráficos correspondentes a esses dados são apresentados na Figura 2.

\subsection{O mercado de câmbio no período analisado}

O período no qual as taxas de câmbio são analisadas se inicia em abril de 1995 e termina em outubro de 2004. A seguir, são apresentadas algumas características do mercado de câmbio brasileiro no período, as quais irão subsidiar as análises a serem feitas na seção 4.

No período analisado, o mercado de câmbio no Brasil era dividido em dois segmentos: o Mercado de Câmbio de Taxas Livres e o Mercado de Câmbio de Taxas Flutuantes.

O Mercado de Câmbio de Taxas Livres é também conhecido como "Comercial" e foi instituído pela Resolução 1.690, de 18/03/1990, do Conselho Monetário Nacional (CMN). Os operadores autorizados a operar nesse mercado são, exclusivamente, bancos comerciais. As operações realizadas nesse segmento 
Tabela 2 - Caracterização das séries de log-retornos e log-volatilidades estudadas

\begin{tabular}{ccc}
\hline & Retornos & Volatilidades \\
\hline Média & 0.0005 & 0.0048 \\
Mediana & 0.0002 & 0.0017 \\
Máximo & 0.0998 & 0.1034 \\
Mínimo & -0.1034 & 0.0000 \\
Desvio-padrão & 0.0095 & 0.0082 \\
Assimetria & 0.4089 & 4.7335 \\
Curtose & 29.6829 & 40.3221 \\
Jarque-Bera & 73699.33 & 153322 \\
Probabilidade & 0 & 0 \\
Observações & 2482 & 2482 \\
\hline
\end{tabular}
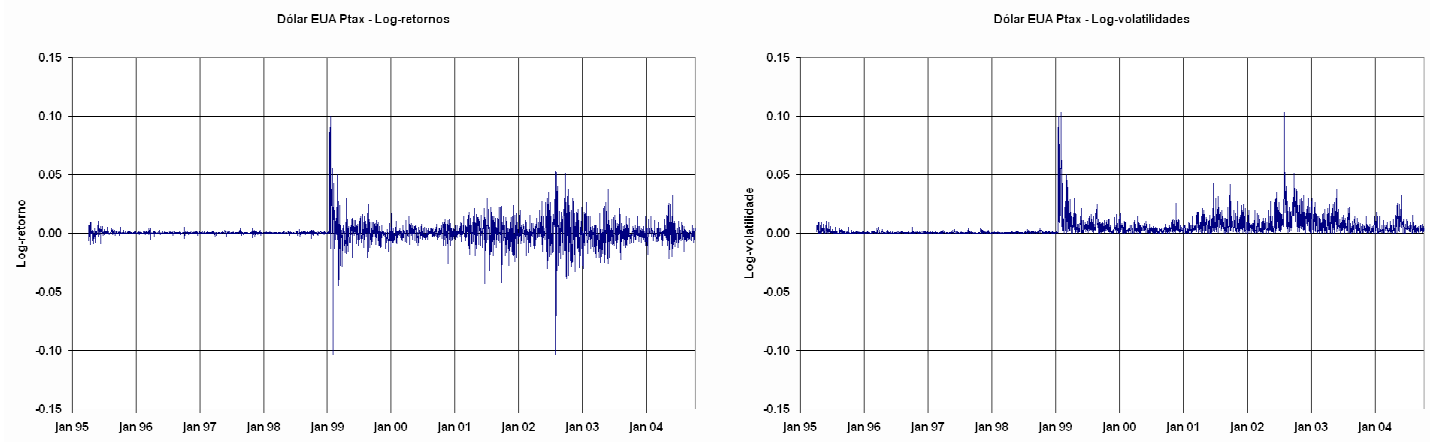

Figura 2 - Séries analisadas: log-retornos e log-volatilidades

são (a) decorrentes de comércio exterior, ou seja, de exportação e de importação; (b) relacionadas às atividades dos governos, nas esferas federal, estadual e municipal; (c) relativas aos investimentos estrangeiros no País e aos empréstimos a residentes sujeitos a registro no Banco Central; e (d) referentes aos pagamentos e recebimentos dos serviços.

Por sua vez, o Mercado de Câmbio de Taxas Flutuantes é também conhecido como "Turismo", e foi instituído pela Resolução 1.552 ,de 22/12/1998, do CMN. Os operadores credenciados a operar nesse mercado são: bancos, entre os quais se incluem os autorizados a operar no Mercado de Câmbio de Taxas Livres; sociedades corretoras, sociedades distribuidoras de títulos e valores mobiliários, sociedades de crédito, financiamento e investimento e meios de hospedagem. Todos esses operadores devem ser credenciados pelo Banco Central. Além das operações relacionadas a turismo, são realizadas nesse mercado transferências, como contribuições a entidades associativas, doações, heranças, aposentadorias e pensões, manutenção de residentes e tratamentos de saúde.

Os agentes do mercado de câmbio, nos dois segmentos, podem assumir posição comprada ou vendida, dentro de limites estabelecidos e monitorados pelo Banco Central. Dentre os bancos autorizados a operar com câmbio, são selecionados semestralmente os chamados dealers de câmbio, que são as instituições com quem a mesa de câmbio do Banco Central opera quando realiza compra e venda no mercado spot. No período analisado, o número desses dealers chegou a sessenta. Em outubro de 2004, eram dezessete os dealers de câmbio.

Entre abril de 1995 e outubro de 2004, estiveram em vigor três regimes cambiais. Inicialmente, 
vigorou o regime cambial com intrabandas. Esse regime esteve em vigor no período de 6/3/1995 a 12/1/1999. No período de 13 a 15/1/1999, as intrabandas foram abolidas, e a partir de 18/1/1999 foi adotado o regime de câmbio flutuante.

No regime de bandas cambiais com intrabandas, as bandas dentro das quais a taxa de câmbio deveria ficar eram definidas pelo Banco Central por meio de Comunicados do Banco Central. No período de vigência desse regime, foram feitos seis desses comunicados. No entanto, o monitoramento da evolução dessas taxas de câmbio era feito dentro de limites bem mais estreitos: as chamadas intrabandas. A taxa de câmbio era mantida dentro dessas intrabandas por meio de operações do Banco Central, realizadas sempre que as taxas de câmbio saíssem dos limites da intrabanda. A partir de 21/6/1995, data em que foi instituído o leilão eletrônico de spread, os limites da intrabanda eram informados ao mercado por meio de parâmetros fornecidos pelo Banco Central para esse tipo de leilão. Desde o início de 1998 até o fim do período de vigência desse regime cambial, foram feitos sete anúncios de intrabanda por mês.

Durante o período de vigência do regime de bandas cambiais com intrabandas, o Banco Central atuava no mercado de câmbio essencialmente por meio de operações diretas junto aos dealers de câmbio e de leilões eletrônicos de compra, venda ou de spread de taxas de câmbio, para cada segmento do mercado de câmbio. A partir do anúncio da moratória russa, em 17 de agosto de 1998, começaram a ocorrer saídas líquidas de divisas no mercado livre, como conseqüência de rumores sobre:

1. possibilidade de mudança de política cambial, mediante desvalorização do real e/ou adoção do câmbio flutuante;

2. imposição de restrição ao fluxo de capitais, para restringir importações e remessas de capitais;

3. possibilidade de declaração unilateral de moratória, em prejuízo dos credores privados, como a Rússia acabara de fazer, e:

4. a expectativa de que o Governo Federal, face ao processo eleitoral então em curso, retardaria a adoção de medidas fortes com a finalidade de reverter o quadro de desconfiança crescente que caracterizava o mercado.

No trimestre de julho a setembro de 1998 , a autoridade monetária vendeu para o mercado US\$ 26,5 bi , e no trimestre de outubro a dezembro de 1998, o Banco Central vendeu mais US\$ 8,5 bi para o mercado, para fazer frente às saídas de recursos associadas a preocupações dos agentes com a possibilidade iminente de mudanças na política cambial. Esses temores se agravaram no início de janeiro de 1999, por conta de:

a) especulações sobre a revisão do acordo com o Fundo Monetário Internacional (FMI), por conta de substancial queda nas reservas cambiais no $2^{\circ}$ semestre de 1998;

b) declaração formal de moratória, por noventa dias, do governo do Estado de Minas Gerais;

c) especulações sobre mudanças no Banco Central e no Ministério da Fazenda;

d) quedas históricas do Índice Bovespa, com influência nas principais bolsas latino-americanas e européias.

e) queda de preço dos títulos soberanos do Brasil e de outros países emergentes;

f) redução drástica da credibilidade do País.

Para defender o regime cambial de bandas, o Banco Central vendeu US\$2,2 bi em 12 de janeiro de 1999, US\$2,0 bi no dia 13 e US\$2,8 bi no dia 14 . 
Em 13/1/1999, o Banco Central divulgou, por meio do Comunicado 6.560, que deixaria de definir e utilizar as intrabandas como limites dentro dos quais as taxas de câmbio deveriam permanecer, dotando o mercado de maior autonomia na definição da taxa. No entanto, o novo regime não conquistou a confiança do mercado, sendo necessário ainda um alto volume de saídas financeiras para manter a taxa de câmbio dentro dos limites da banda maior. Como conseqüência, em 15/1, o Banco Central emitiu o Comunicado 6.563, suspendendo temporariamente o regime de bandas e, em 18/1 (segunda-feira), o Banco Central emitiu o Comunicado 6.565, informando que, a partir daquela data, deixaria que o mercado definisse a taxa de câmbio nos segmentos livre e flutuante, realizando operações, ocasionalmente, com o objetivo de reduzir a volatilidade. Alguns dias depois, também foram adotadas medidas para disciplinar a posição de câmbio dos agentes do mercado. No último dia útil de janeiro, o dólar dos EUA tinha valorizado 63,7\% com relação a 12/1/1999 e 64,08\% em janeiro. Depois que o regime de câmbio passou para flutuante, além da mudança nos objetivos de suas operações, o Banco Central mudou a maneira de atuar no mercado. Os leilões eletrônicos de câmbio não foram mais utilizados e foram estabelecidas restrições nos montantes de divisas que o Banco Central poderia vender. Mais tarde, em 2002, ficou estabelecido que o governo não mais emitiria títulos públicos com cláusula cambial com finalidade diferente de rolagem de dívida. Essa restrição tinha a finalidade de reduzir a exposição cambial da dívida interna brasileira.

Depois que o regime de câmbio mudou, ainda ocorreram momentos de turbulência no mercado. Em outubro de 2001, ocorreu uma ligeira valorização do dólar, como resultado do atentado terrorista de 11 de setembro e das incertezas quanto aos desenvolvimentos econômicos na Argentina. No início de novembro, o mercado começou a dissociar os números de risco do Brasil e da Argentina, entendendo que o Brasil apresentava fundamentos econômicos mais sólidos, que diferenciavam sua situação da situação da Argentina, o que permitiu que o preço do dólar caísse. No trimestre final de 2001, o Banco Central vendeu US\$3 bi. Em janeiro, o dólar valorizou até 8 de fevereiro, que foi o último dia de câmbio fixo na Argentina. Em 11 de fevereiro de 2002, quando a Argentina passou a adotar o regime de câmbio flutuante, o dólar voltou a desvalorizar, atingindo o nível do início do ano.

\section{RESULTADOS EMPÍRICOS}

Os cálculos dos expoentes de Hurst ao longo do tempo foram feitos, de acordo com a metodologia apresentada, obtendo-se os gráficos da Figura 3 para retornos e os da Figura 5 para volatilidades. Esses gráficos apresentam valores com intervalo de confiança de $95 \%$ para $\mathrm{H}$, conforme o modelo explicitado pela equação (7), estimados pelo método dos mínimos quadrados ordinários. Os valores atribuídos ao expoente de Hurst em uma data representam o resultado do cálculo feito para uma janela de dados de 504 observações que se inicia nessa data. Isso pode ser visto na figura 4. A amostra foi embaralhada dentro de blocos contíguos de dez observações para eliminar os efeitos que a possível ocorrência de autocorrelações de curto prazo poderia ter, por esse motivo, não foi feita filtragem dos retornos e volatilidades com relação a dependências relacionadas a dias-de-semana. As séries de expoentes de Hurst ao longo do tempo obtidas para retornos e volatilidades foram caracterizadas estatisticamente, sendo os resultados apresentados na Tabela 3. Para essas séries, é calculada a estatística de Jarque-Bera. Nos casos das séries de expoentes de Hurst calculadas para retornos e volatilidades das taxas de câmbio, o p-valor obtido permite rejeitar a hipótese nula de normalidade dessas séries ao nível de $1 \%$, o que indica que esses expoentes realmente variam com o tempo.

O gráfico dos expoentes de Hurst ao longo do tempo obtidos para os retornos (Figura 3) apresenta duas regiões distintas: na primeira, que se estende até o início de 1999, o expoente de Hurst, depois de apresentar um período levemente descendente, sobe a valores acima de 0,65. Na segunda, a partir de 1999, o expoente de Hurst se mantém aproximadamente constante, na faixa dos 0,6.

A compreensão desse comportamento requer duas informações adicionais: a série de retornos, como apresentada na Figura 2, e a informação de que a amostra utilizada no cálculo tem comprimento finito. 
Tabela 3 - Caracterização das séries de Expoentes de Hurst calculados

\begin{tabular}{ccc}
\hline & $H_{\text {Retornos }}$ & $H_{\text {Volatilidades }}$ \\
\hline Média & 0.5685 & 0.7353 \\
Mediana & 0.5866 & 0.7470 \\
Máximo & 0.6907 & 0.8171 \\
Mínimo & 0.4248 & 0.6197 \\
Desvio-padrão & 0.0687 & 0.0484 \\
Assimetria & -0.4808 & -0.8875 \\
Curtose & 2.2058 & 2.7657 \\
Jarque-Bera & 128.2644 & 264.3163 \\
Probabilidade & 0 & 0 \\
Observações & 1979 & 1979 \\
\hline
\end{tabular}

O fato de a amostra utilizada nos cálculos ser finita causa uma distorção no resultado do cálculo do expoente de Hurst. Para amostras com ciclos de periodicidade e amplitude regulares, uma amostra de comprimento tendendo a infinito produz como resultado um expoente de Hurst tendendo a zero. À medida que o comprimento da amostra utilizada no cálculo diminui, o expoente de Hurst vai aumentando (a estatística $R / S$ tem valor finito e seu valor permanece aproximadamente constante ao se diminuir o comprimento da amostra, até o comprimento de 1 ciclo), chegando a 0,5 e, se o comprimento da amostra for igual ao período do ciclo, o expoente de Hurst chegará a um valor próximo de 1 . 0 fato de se utilizar amostra finita introduz um viés por cima no expoente de Hurst. Uma análise da série de retornos utilizada como base nos cálculos do expoente de Hurst mostra uma amostra dividida em dois períodos distintos, que correspondem aos dois regimes de câmbio principais, que estiveram em vigor ao longo do período amostral. Até o início de 1999, vigorou o regime cambial de bandas com intrabandas. No início da amostra, durante o ano de 1995, houve um período de maior instabilidade, como reflexo da crise do México, em que foi difícil manter a taxa de câmbio dentro de limites mais estreitos. Essa dificuldade resultou na existência de oscilações muito variáveis no tempo, que depois se tornaram menores e mais regulares.

Isso explica a queda no expoente de Hurst, verificada no início da amostra. Superado esse período inicial, até o final do período do regime cambial de bandas com minibandas, os retornos oscilaram de maneira regular e periódica, o que resultou em antipersistência ${ }^{5}$, cuja visibilidade seria aumentada caso se utilizassem janelas de comprimento maior. Os expoentes calculados a partir de 1997 começaram a subir, como conseqüência da inclusão, na janela de cálculo, dos retornos ocorridos durante e a partir da crise que levou à adoção do regime de câmbio flutuante, em janeiro de 1999, conforme é mostrado na Figura 4. De janeiro a março de 1999, ocorreram grandes oscilações nos preços, retornos e volatilidades, que resultaram em aumento do expoente de Hurst calculado a partir de janelas que continham esse período. A partir do final de março de 1999, quando as grandes oscilações nos preços tinham desaparecido, notou-se a diminuição do expoente de Hurst e sua manutenção no patamar de 0,6 até o final da amostra (Figura 4). No período do regime de bandas cambiais, a antipersistência verificada não se deve à ocorrência de crises ou às operações que o Banco Central realizava, mas ao objetivo buscado com essas operações: a manutenção da taxa de câmbio em faixas estreitas, de forma que, a cada oscilação mais abrupta, de subida ou descida da taxa, correspondia uma ação do Banco Central para reverter o quadro. Essa maneira de atuar deu origem a um padrão cíclico irregular, mas de amplitude aproximadamente constante. A ocorrência de crises, com a da Ásia ou da Rússia, apesar de exigir esforço adicional do Banco Central, não provocou alterações nas medidas de memória de longo prazo dos retornos, que

\footnotetext{
${ }^{5}$ Série temporal antipersistente é a série que tende a apresentar, em um período qualquer, movimento contrário ao apresentado no período anterior, e que possui memória de longo prazo com expoente de Hurst no intervalo $0<\mathrm{H}<0,5$.
} 


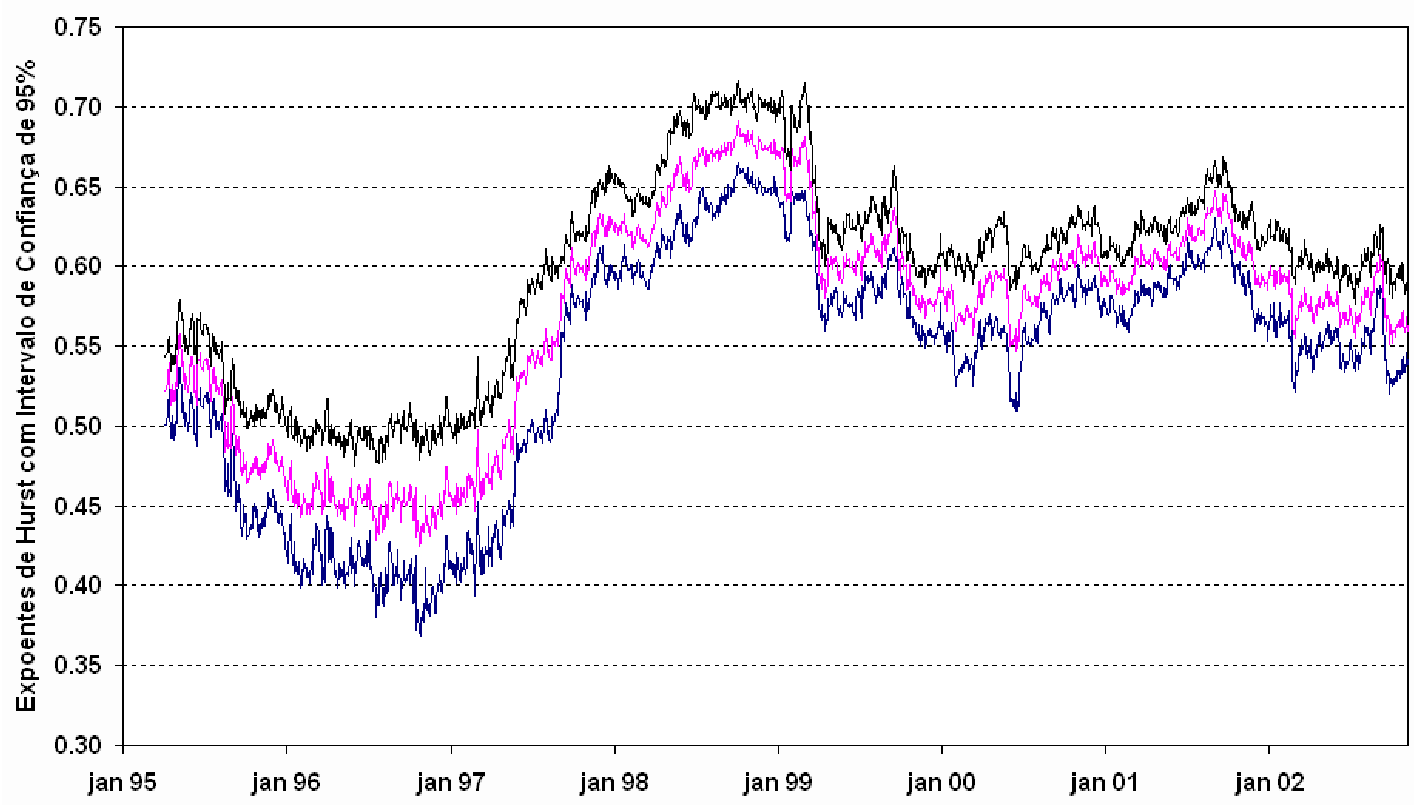

Figura 3 - Expoentes de Hurst ao longo do tempo - Retornos

continuaram no mesmo patamar. Somente quando ocorreu o ataque especulativo de 12 a 14 de janeiro de 1999, que resultou na venda líquida de US\$7,0 bi pelo Banco Central e na decisão de abandonar o regime cambial de bandas, houve uma mudança efetiva nos valores de memória de longo prazo calculados para a série. No início da crise, os bancos decidiram assumir posição fortemente comprada, o que elevou muito a taxa de câmbio. Passada a instabilidade inicial, essa posição foi abandonada, o que permitiu que a taxa recuasse em retorno a um patamar mais alto do que o existente no início da crise. Uma das causas do aumento da memória de longo prazo verificado nos cálculos para janelas de dados envolvendo esse período de crise foi justamente a ocorrência de grandes oscilações nos retornos e volatilidades do período.

O fato de o Banco Central não mais ter um compromisso com um nível para a taxa de câmbio foi uma mudança estrutural importante. Além dessa mudança, contribuiu para a mudança estrutural o acordo com o FMI que estabelecia uma limitação no montante mensal e individual das operações do Banco Central. Como resultado dessa nova sistemática, a taxa de câmbio pôde variar de maneira muito mais livre em momentos como a crise da Argentina, no início de 2002, e as eleições presidenciais no Brasil, no final desse ano. Apesar das grandes oscilações na taxa nesse período, não se verificaram alterações significativas na memória de longo prazo associada a ela, indicando que a estrutura do mercado não variou significativamente, do ponto de vista dos processos geradores de taxas de câmbio no período, embora esses processos estivessem sujeitos a mudanças nas expectativas dos agentes do mercado, aqui entendidas como fatores exógenos.

No caso das volatilidades, observa-se um comportamento semelhante ao observado para os retornos, isto é, a memória de longo prazo observada para as volatilidades no período do regime cambial de bandas era menor do que a observada a partir da liberação do câmbio. Também se nota, no período a partir de 1999, a constância da memória de longo prazo em torno de um valor, que nesse caso é 0,75 . 


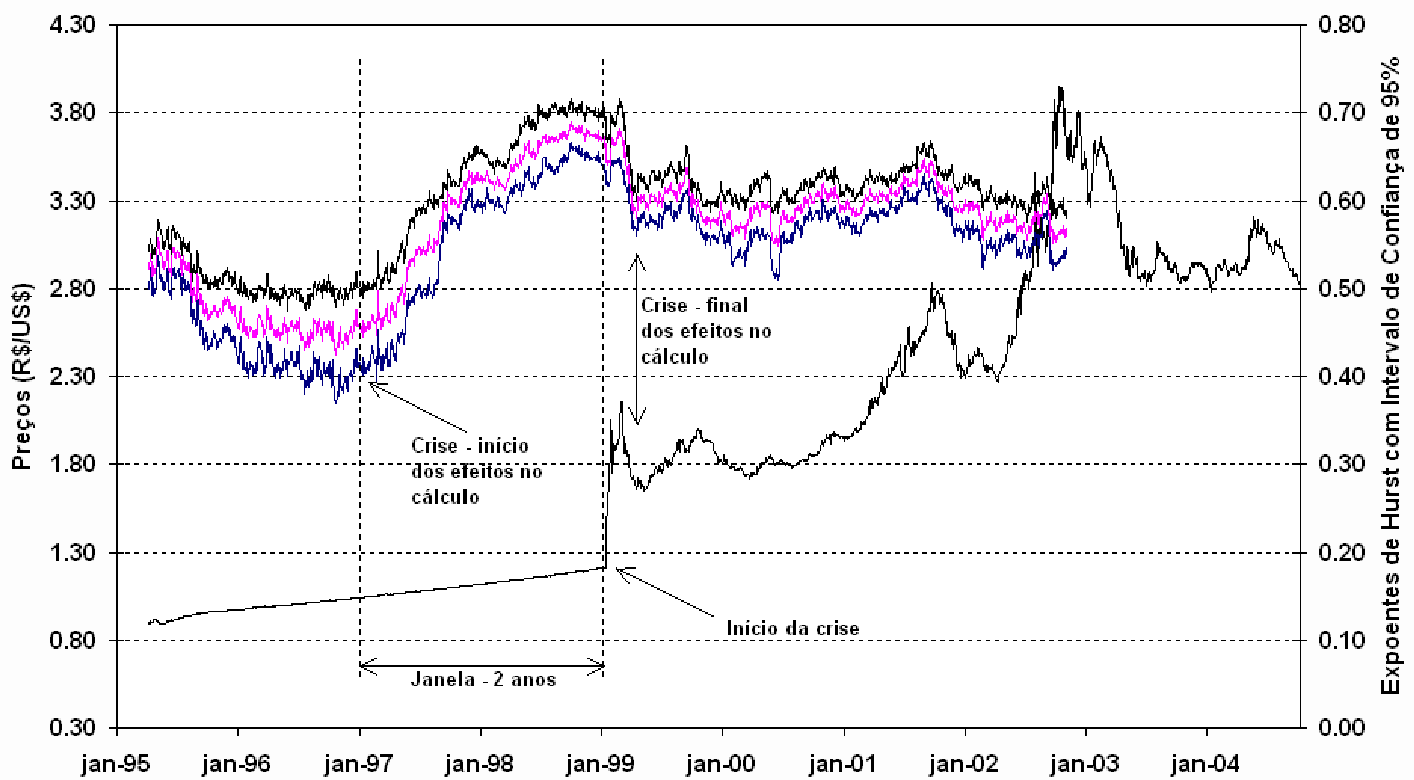

Figura 4 - Reflexo da mudança no regime de câmbio na

Além disso, no período inicial de 1995, logo após a crise do México, observa-se uma queda substancial. A diferença mais evidente entre os gráficos de memória de longo prazo de retornos e volatilidades está no fato de a memória de longo prazo das volatilidades já se encontrar em um patamar alto - de 0,80 -, por ocasião da crise da Ásia e no período da crise da Rússia, indicando que a instabilidade que essas crises provocaram no padrão oscilatório das taxas de câmbio, ainda que de amplitude muito menor do que a verificada no período após a adoção do regime de câmbio flutuante, foi semelhante do ponto de vista da memória de longo prazo. Essa semelhança é possível, pois a estatística $R / S$ na qual se baseia o cálculo do expoente de Hurst extrai a média da série no período " $\tau$ " da amostra e a divide pelo desvio-padrão, o que faz com que a amplitude das oscilações em si não seja importante, mas apenas as variações nas médias e nas amplitudes ao longo do período " $\tau$ " da amostra.

\section{CONCLUSÃO}

Os cálculos feitos para o expoente de Hurst da taxa de câmbio Real $\times$ Dólar no Brasil, para retornos, evidenciaram uma associação entre o regime cambial em vigor e a medida de memória de longo prazo obtida. A medida de memória de longo prazo obtida para o período de regime cambial de bandas indicou antipersistência. Além disso, encontrou-se evidência de que o regime de bandas manteve as taxas de câmbio do País isoladas das crises que ocorreram no período, como as crises da Ásia e da Rússia.

Esses eventos, no entanto, afetaram as volatilidades da taxa de câmbio. Após a adoção do regime de câmbio flutuante, foi possível verificar que, tanto no caso dos retornos quanto no caso das volatilidades, apesar das grandes variações de taxa ocorridas no período, a estrutura do processo que gerou essas taxas não sofreu modificações significativas, o que pode ser constatado a partir da verificação de que a medida de memória de longo prazo feita para esse período se mantém em torno de um valor fixo. 
Série: Dólar EUA Ptax I Volatilidades

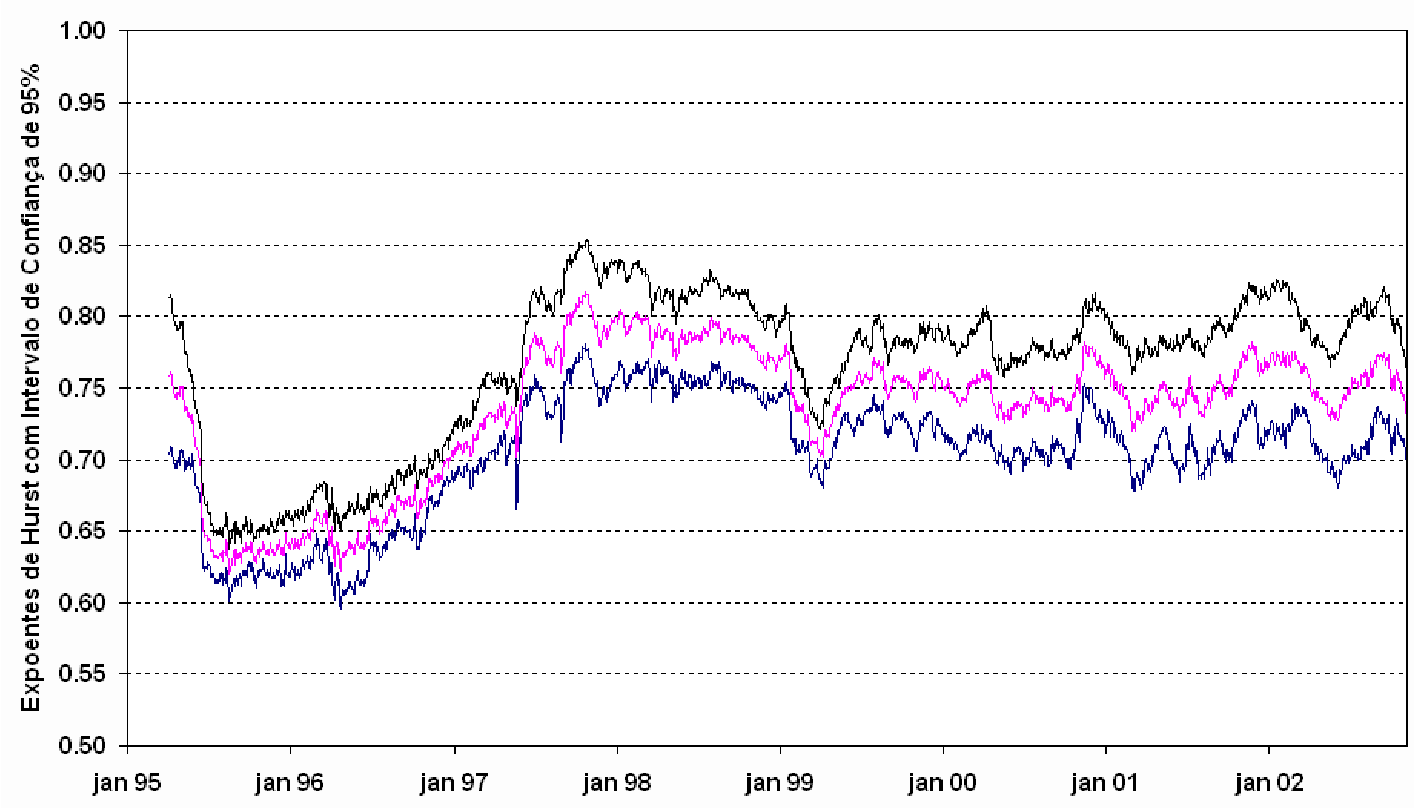

Figura 5 - Expoentes de Hurst ao longo do tempo - Volatilidades

Em consonância com outros trabalhos, a medida de memória de longo prazo obtida para as volatilidades é maior do que a obtida para os retornos, sendo ambas indicativas de memória de longo prazo no período do regime de câmbio flutuante. A mudança do regime de câmbio foi uma quebra estrutural que se refletiu na memória de longo prazo calculada para os retornos.

Finalmente, foi possível obter resultados qualitativos que podem ter utilidade na realização de previsões, para uma janela de cálculo de comprimento finito e amostra de dados não estacionária.

\section{Referências Bibliográficas}

Análise do Mercado de Câmbio. (2005). Brasília: Banco Central do Brasil. Relatório Trimestral. Edições desde o $3^{\circ}$ trimestre de 1998 até o $4^{\circ}$ trimestre de 2002. Disponíveis em http://www.bcb.gov.br/. Acesso em 26 maio 2005.

Bollerslev, T., Baillie, R., \& Mikkelsen, H. (1996). Fractionally integrated generalized autoregressive conditional heteroskedasticity. Journal of Econometrics, 74:3-30.

Cajueiro, D. \& Tabak, B. (2004a). Evidence of long range dependence in Asian equity markets: the role of liquidity and market restrictions. Physica A, 342(3-4):656-664.

Cajueiro, D. \& Tabak, B. (2004b). Ranking Efficiency for Emerging Markets. Chaos, Solitons and Fractals, 22(2):349-352.

Cajueiro, D. \& Tabak, B. (2004c). The Hurst exponent over time: testing the assertion that emerging markets are becoming more efficient. Physica A, 336(3-4):521-537. 
Cajueiro, D. \& Tabak, B. (2005a). Possible causes of long-range dependence in the Brazilian stock market. Physica A, 345(3-4):635-645.

Cajueiro, D. \& Tabak, B. (2005b). Ranking Efficiency for Emerging Markets II. Chaos, Solitons and Fractals, 23:671.

Cajueiro, D. \& Tabak, B. (2005c). Testing for time-varying long-range dependence in volatility for emerging markets. Physica A, 346(3-4):577-588.

Cajueiro, D. \& Tabak, B. (2005d). The rescaled variance statistic and the determination of the Hurst exponent. Mathematics and Computers in Simulation, 70(3):172-179.

Couillard, M. \& Davison, M. (2005). A comment on measuring the Hurst exponent of financial time series. Physica A, 348:404-418.

Crato, N. \& Ray, B. (2000). Memory in returns and volatilities of futures' contracts. Journal of Futures Markets, 20(6):525-543.

Erramilli, A., Narayan, O., \& Willinger, W. (1996). Experimental queueing analysis with long-range dependent packet traffic. IEEE/ACM Transactions on Networking (TON), 4(2):209-223.

Feder, J. (1988). Fractals. Plenum Press New York.

Geweke, J. \& Porter-Hudak, S. (1983). The Estimation and Application of Long Memory Time Series Models. Journal of TIme Seires Analysis, 4:221-238.

Giraitis, L., Kokoszka, P., Leipus, R., \& TeyssiÂere, G. (2003). Rescaled variance and related tests for long memory in volatility and levels. Journal of Econometrics, 112:265-294.

Granger, C. W. J. \& Joyeux, R. (1980). An introduction to long-range time series models and fractional differencing. Journal of Time Series Analysis, 4:221-238.

Hosking, J. (1981). Fractional Differencing. Biometrika, 68(1):165-176.

Hurst, H. (1951). Long term storage capacity of reservoirs. Transactions of thr American SOciety of Civil Engineers, 116:770-799.

Laurini, M. \& Portugal, M. (2004). Long Memory int the R\$/US\$ Exchange Rate: A Robust Analysis. Brazilian Review of Econometrics, 24(1):109-147.

Lo, A. (1991). Long-Term Memory in Stock Market Prices. Econometrica, 59(5):1279-1313.

Mandelbrot, B. (1971). When Can Price be Arbitraged Efficiently? A Limit to the Validity of the Random Walk and Martingale Models. The Review of Economics and Statistics, 53(3):225-236.

Mandelbrot, B. (1972). Statistical methodology for non-periodic cycles: from the covariance to R/S analysis. Annals of Economic and Social Measurement, 1(3):259-290.

Mandelbrot, B. (1975). Limit theorems on the self-normalized range for weakly and strongly dependent processes. Probability Theory and Related Fields, 31(4):271-285.

Mandelbrot, B. (1982). The fractal geometry of nature: San Francisco. WH Freeman and Co.

Mandelbrot, B. \& Wallis, J. (1969a). Computer experiments with fractional Gaussian noises. Parts 1,2,3. Water Resources Research, 5(1):228-267. 
Mandelbrot, B. \& Wallis, J. (1969b). Robustness of the rescaled range R/S in the measurement of noncyclic long run statistical dependence. Water Resources Research, 5(5):967-988.

Moreira, J., Silva, J., \& Kamphorst, S. (1994). On the fractal dimension of self-affine profiles. Journal of Physics A: Mathematical and General, 27(24):8079-8089.

Muniandy, S., Lim, S., \& Murugan, R. (2001). Inhomogeneous scaling behaviors in Malaysian foreign currency exchange rates. Physica A, 301(1):407-428.

Peng, C., Buldyrev, S., Havlin, S., Simons, M., Stanley, H., \& Goldberger, A. (1994). Mosaic organization of DNA nucleotides. Physical Review E, 49(2):1685-1689.

Robinson, P. (1995). Log-Periodogram Regression of Time Series with Long Range Dependence. Annals of Statistics, 23(3):1048-1072.

Taqqu, M., Teverovsky, V., \& Willinger, W. (1995). Estimators for long-range dependence: An empirical study. Fractals, 3(4):785-798.

Teverovsky, V., Taqqu, M., \& Willinger, W. (1999). A critical look at Lo's modified R/S statistic. Journal of Statistical Planning and Inference, 80(1):211-227.

Wallis, J. \& Matalas, N. (1970). Small Sample Properties Of H And K-Estimators Of The Hurst Coefficient H. Water Resource Research, 6:1583-1594. 